\title{
Behavioral, neurochemical and morphological changes induced by the overexpression of munc18-1a in brain of mice: relevance to schizophrenia
}

\author{
L Urigüen ${ }^{1,2,3}$, I Gil-Pisa ${ }^{1,2}$, E Munarriz-Cuezva ${ }^{1,2}$, E Berrocoso ${ }^{1,4}$, J Pascau ${ }^{1,5}$, ML Soto-Montenegro $^{1,5}$, A Gutiérrez-Adán 6 , B Pintado ${ }^{6}$, \\ JLM Madrigal $\left.\right|^{1,7}$, E Castro ${ }^{1,8}$, P Sánchez-Blázquez ${ }^{1,9}$, JE Ortega ${ }^{1,2,3}$, MJ Guerrero ${ }^{10}$, M Ferrer-Alcon ${ }^{10}$, JA García-Sevilla ${ }^{11}$, JA Micó ${ }^{1,4}$, \\ M Desco ${ }^{1,5,12}$, JC Leza ${ }^{1,7}$, Á Pazos ${ }^{1,8}$, J Garzón ${ }^{1,9}$ and JJ Meana ${ }^{1,2,3}$
}

Overexpression of the mammalian homolog of the unc-18 gene (munc18-1) has been described in the brain of subjects with schizophrenia. Munc18-1 protein is involved in membrane fusion processes, exocytosis and neurotransmitter release. A transgenic mouse strain that overexpresses the protein isoform munc18-1a in the brain was characterized. This animal displays several schizophrenia-related behaviors, supersensitivity to hallucinogenic drugs and deficits in prepulse inhibition that reverse after antipsychotic treatment. Relevant brain areas (that is, cortex and striatum) exhibit reduced expression of dopamine $D_{1}$ receptors and dopamine transporters together with enhanced amphetamine-induced in vivo dopamine release. Magnetic resonance imaging demonstrates decreased gray matter volume in the transgenic animal. In conclusion, the mouse overexpressing brain munc18-1a represents a new valid animal model that resembles functional and structural abnormalities in patients with schizophrenia. The animal could provide valuable insights into phenotypic aspects of this psychiatric disorder. Translational Psychiatry (2013) 3, e221; doi:10.1038/tp.2012.149; published online 22 January 2013

\section{Introduction}

Schizophrenia is a chronic and disabling psychiatric disorder that affects about $1 \%$ of the population worldwide. Symptoms of schizophrenia include positive, negative and cognitive deficits. ${ }^{1}$ The hypothesis that dopaminergic mechanisms play an important role in the pathophysiology of schizophrenia has been one of the most accepted ideas about the disease. ${ }^{2}$ In addition, in vivo neuroimaging studies have demonstrated reduced gray matter volume in patients with schizophrenia. ${ }^{3,4}$ Although the processes involved in the development of schizophrenia remain unclear, it has been suggested that dysfunctions in neuroexocytosis could be a key point in the pathophysiology of the disorder. ${ }^{5}$

The proteins responsible for the mechanism of membrane fusion and neurotransmitter release (SNAP-25, syntaxin-1A and synaptobrevin) form the SNARE (soluble $\mathrm{N}$-ethylmaleimide-sensitive factor attachment protein receptors). ${ }^{6}$ Munc18-1 interacts with syntaxin-1A and other SNARE proteins, and influences all of the steps leading to exocytosis, including vesicle recruitment, docking and membrane fusion. $^{7,8}$ It has been proposed that abnormalities of munc18-1 and/or SNARE proteins might participate in the pathogenesis of schizophrenia underlying dysfunctional neuroexocytosis. ${ }^{5,9}$ Thus, proteomic studies in postmortem prefrontal cortex of subjects with schizophrenia have shown increases of munc18-1 in membrane microdomains of the gray matter. ${ }^{10}$ Moreover, antipsychotic treatment seems to be associated with a lower content of key proteins of the exocytotic machinery, which could results in a destabilization/ impairment of neuroexocytosis. ${ }^{11}$

Alternative splicing of the gene coding for munc18-1 results in two variants, the long isoform (munc18-1a, brain and retina) and the ubiquitous short isoform (munc18-1b). ${ }^{12,13}$

A wide number of animal models of schizophrenia have been developed ${ }^{14}$ in an effort to reproduce one or more of the symptoms observed in humans. Animal models represent a very useful tool for the study of the mechanisms underlying schizophrenia-like behaviors and for the evaluation of the therapeutic efficacy of antipsychotics. ${ }^{14,15}$ In this context, a transgenic mouse overexpressing the isoform munc18-1a protein was developed to assess whether abnormalities of exocytosis machinery could lead to emergence of schizophrenic phenotypes.

\section{Materials and methods}

All animal studies were performed in accordance with the European Directive for the Protection of Vertebrate Animals

\footnotetext{
${ }^{1}$ Centro de Investigación Biomédica en Red de Salud Mental CIBERSAM, Spain; ${ }^{2}$ Department of Pharmacology, University of the Basque Country UPV/EHU Leioa, Bizkaia, Spain; ${ }^{3}$ BioCruces Health Research Institute, Bizkaia, Spain; ${ }^{4}$ Department of Neuroscience, University of Cádiz, Cádiz, Spain; ${ }^{5}$ Department of Experimental Medicine and Surgery, General Hospital Gregorio Marañón, Madrid, Spain; ${ }^{6}$ Department of Animal Reproduction, INIA, Madrid, Spain; ${ }^{7}$ Department of Pharmacology, Faculty of Medicine, Complutense University, Madrid, Spain; ${ }^{8}$ Department of Physiology and Pharmacology, University of Cantabria, Institute of Biomedicine and Biotechnology of Cantabria IBBTEC), Santander, Spain; ${ }^{9}$ Cajal Institute, CSIC, Madrid, Spain; ${ }^{10}$ Brainco Biopharma, SL, Derio, Bizkaia, Spain; ${ }^{11}$ Neuropharmacology Laboratory, IUNICS, University of Balearic Islands, Palma de Mallorca and Redes Temáticas de Investigación Cooperativa en Salud, Red de Trastornos Adictivos (RETICS-RTA), Spain and ${ }^{12}$ Department of Bioengineering and Aerospace Engineering, Carlos III University, Madrid, Spain

Correspondence: Dr JJ Meana, Department of Pharmacology, University of the Basque Country (UPV/EHU), Leioa, Bizkaia 48940, Spain.

E-mail: javier.meana@ehu.es

Keywords: Munc18-1a; PPI; schizophrenia; SNARE
} 
used for experimental and Other Scientific Purposes (European Union Directive \#86/606/EEC). All animals were 3- to 6-month-old males.

Generation of the transgenic animal. Animals were created by pronuclear microinjection to C57BL6/CBA embryos. ${ }^{16}$ A sequence of 2848 nucleotides from the GTRAP3-18 promoter was amplified by PCR and cloned using the Kpnl-Sall sites, in the right anterior position of human Munc18-1a cDNA (NIH_MGC_94), in the pCMVSport6 commercial vector. The construct was digested and the transgene microinjected in mouse embryo. This transgene fragment, in addition to the GTRAP3-18 promoter and the cDNA sequence for Munc18-1a (clone IRAVp968D0260D, www.imagenes-bio.de), contained a SV40 polyadenylation site for mRNA stabilization. Four transgenic founders were produced and in three of them (lines L3, L7 and L8), the transgene was Mendelian fashion transmitted. Immunoblotting analysis showed that line L8 did not display increased expression of munc18-1a (Supplementary Figure S1) while line L3 presented impaired breeding. After initial animal characterization of the three lines (data not shown), line L7 (termed as Munc18-OE) was selected for further assays.

Quantitative real-time PCR. QRT-PCR was performed with a StepOne ${ }^{\mathrm{TM}}$ thermocycler using a TaqMan $^{\mathrm{TM}}$ assay (Applied Biosystems, Foster City, CA, USA) specifically designed for recognizing munc18-1a. Data were analyzed with the StepOne ${ }^{\mathrm{TM}}$ Software v2.1 (Applied Biosystems). This procedure has been described earlier. ${ }^{17}$

In situ hybridization. Sections $(20 \mu \mathrm{m})$ were thaw mounted onto slides and pre-treated using a protocol previously described. ${ }^{18}$ An oligonucleotide complementary to mouse munc18-1a mRNA was labeled with $\left[{ }^{35} \mathrm{~S}\right] \mathrm{dATP}$. After incubation with the labeled probe, slides were washed, air dried and exposed to films together with ${ }^{14} \mathrm{C}$ microscales at $-20^{\circ} \mathrm{C}$ for 4 weeks. Experiments were quantified using Scion Image system (Scion Corporation, Frederick, MD, USA).

Immunoblotting. Immunoblotting procedures were carried out as described earlier. ${ }^{19}$ Briefly, electrophoresis was carried out and membranes were incubated with specific antibodies (Supplementary Table S1). Later, the membranes were incubated with the respective secondary antibodies, visualized on X-ray film by chemiluminescence and quantified by densitometry.

Immunofluorescence. The brains were fixed by transcardial perfusion (4\% paraformaldehyde) and cryoprotected in $30 \%$ sucrose. The sections $(30 \mu \mathrm{m})$ were blocked and incubated at $4{ }^{\circ} \mathrm{C}$ with anti-munc18-1a antibody. Sections were washed and incubated with the secondary antibody. Fluorescence microscopic analyses were performed with a Nikon microscope (Kanagawa, Japan). Twenty different fluorescence images were carried out in each mouse. Gray intensities in arbitrary units were measured in each image.
Novelty-induced hyperactivity. Mice were placed individually in the center of an open field and spontaneous locomotion was evaluated for $105 \mathrm{~min}$ with a computerized video-tracking system (SMART, PanLab, Spain).

DOI-induced head-twitch. Mice were injected with $1 \mathrm{mg} \mathrm{kg}^{-1}$ i.p. of DOI (2,5-dimethoxy-4-iodoamphetamine) and, $5 \mathrm{~min}$ after injection, recorded for $10 \mathrm{~min} .{ }^{20}$ Subsequent head-twitch response (HTR) was scored.

Elevated plus maze. Mice were individually placed in the maze and time spent in open-arms and number of entries to open-arms were evaluated for $5 \mathrm{~min}^{21}$

Social interaction. Pairs of mice from different home-cages were placed together and the time that mice socially interact was measured for $5 \mathrm{~min}^{21}$

Novel object recognition test. Animals were given two sample sessions and one test trial. In sample 1, the animal was allowed $5 \mathrm{~min}$ to explore four identical novel objects (object $\mathrm{A}$ ) arranged in a triangular shape. In sample 2, the animal explored four new identical objects (object B) arranged in a rectangular shape. Finally, the animal was given a test trial in which two copies of object $B$ were placed as in sample 2, one copy of object $A$ was placed as it had been in sample 1, and one copy of object A was newly located. Exploration was recorded and scored for investigation time of each object in both sample and test sessions.

Prepulse inhibition of the startle reflex. Mice were placed in a startle chamber (PanLab, Spain) and then subjected to a pseudo randomized combination of: (1) 10 pulse-alone trials consisted of a single white noise burst; (2) 10 prepulse-alone trials for each prepulse intensity; (3) 10 prepulse-pulse trials for each prepulse intensity; (4) 10 presentations of no stimulus trial, in which only the background noise was presented. Prepulse inhibition (PPI) was calculated as a percentage score: $\% \mathrm{PPI}=[100-($ startle response to prepulse + pulse trial)/(startle response to pulse-alone trial) $] \times 100$.

Amphetamine-induced behavioral sensitization. Mice were injected with amphetamine $\left(5 \mathrm{mg} \mathrm{kg}^{-1}\right.$, i.p.) and spontaneous locomotion was evaluated with a computerized video-tracking system (SMART, PanLab) for 105 min after injection.

In vivo microdialysis and chromatographic analysis. Animals were anesthetized and placed in a Kopf stereotaxic frame. Intracerebral probes (Cuprophan $2 \mathrm{~mm}$ membrane) were implanted in the striatum (coordinates AP $+1.2 \mathrm{~mm}$, $\mathrm{L}+1.5 \mathrm{~mm}$, DV $-3.2 \mathrm{~mm}$ ) or in the prefrontal cortex $(\mathrm{AP}+2.0 \mathrm{~mm}, \mathrm{~L}+0.3 \mathrm{~mm}, \mathrm{DV}-3.4 \mathrm{~mm})^{22}$ and fixed to the skull. The next day, mice were connected to a fraction collection system for freely moving animals. ${ }^{23}$ Basal values were obtained by no-net-flux method and response to amphetamine $\left(5 \mathrm{mg} \mathrm{kg}^{-1}\right.$ i.p.) was calculated over basal values. Dopamine (DA) concentrations were measured by HPLC coupled to an electrochemical detector. 
Caspase-3. Caspase-3 activity was measured by cleaving the selective substrate Ac-Asp-Glu-Val-Asp-AFC (Biomol International, Plymouth Meeting, PA, USA). Brain cortex was homogenized and centrifuged, and samples were incubated in a total volume of $100 \mu \mathrm{l}$ comprised of $80 \mu \mathrm{l}$ detection buffer. After incubation, cleavage of the substrate was detected measuring fluorescence with a Fluoroscan Ascent reader (Labsystems, Waltham, MA, USA).

Lipid peroxidation. Brain cortex was sonicated and deproteinized with $40 \%$ trichloroacetic acid and $5 \mathrm{M} \mathrm{HCl}$, followed by the addition of $2 \%$ thiobarbituric acid in $0.5 \mathrm{M} \mathrm{NaOH}$. The reaction mixture was heated at $90^{\circ} \mathrm{C}$ for $15 \mathrm{~min}$ and centrifuged at $12000 \times \mathrm{g}$ for $10 \mathrm{~min}$. The pink chromogen was measured at $532 \mathrm{~nm}$ in a Beckman DU-7500 spectrophotometer (Beckman Coulter, CA, USA).

Volumetric study of gray matter. Animals were anesthetized with sevoflurane (4\% for induction and $2 \%$ for maintenance in $100 \% \mathrm{O}_{2}$ ). Images were acquired with a Bruker $7 /$ 210 USR MRI scanner (Bruker Instruments, Billerica, MA, USA). A T2-weighted spin echo sequence was acquired with the following parameters: $256 \times 256$ matrix size covering a field of view of $20 \mathrm{~mm}$, repetition time $3100 \mathrm{~s}$, echo time $35 \mathrm{~ms}, 37$ slices with slice thickness $0.4 \mathrm{~mm}$. Cerebral gray matter differences between groups were studied applying VBM (voxel-based morphometry) methodology with SPM5 software package (Statistical Parametric Mapping, Wellcome Department of Cognitive Neurology, London, UK). Tissue probabilities maps, created with wild-type (WT) and R6/2 transgenic c57 mice, were provided by the author of the spmmouse extension. ${ }^{24}$ VBM statistical analysis consisted of a Student's $t$-test, with the gray matter volume as a covariate, and FDR (false discovery rate) correction for multiple comparisons. Significant results were accepted for $P<0.01$ (corrected for FDR) and a minimum cluster size of 200 voxels.

Data analyses and statistics. Data were analyzed with GraphPad Prism ${ }^{\mathrm{TM}} 4.0$ (GraphPad Software, San Diego, CA, USA), except for volumetric assay of gray matter. Results are expressed as mean \pm s.e.m. values. Data were analyzed by using unpaired Student's $t$-test, one-way or two-way analysis of variance tests followed by Bonferroni post hoc test for multiple comparisons. The level of significance was set to $P<0.05$. All tests were two tailed.

\section{Results}

Selective overexpression of munc18-1a in the brain of transgenic mice. Munc18-1a expression was driven by the GTRAP3-18 promoter, which regulates EAAT3, a neuronal product expressed in glutamatergic and GABAergic neurons. ${ }^{25,26}$ Increased expression of munc18-1a was observed in the brain cortex of the transgenic Munc18-OE mice by QRT-PCR (Figure 1a). An anatomical distribution of the mRNA encoding for munc18-1a protein was observed by in situ hybridization through different structures of the mouse brain. The specificity of munc18-1a mRNA labeling was demonstrated by the displacement with unlabeled oligonucleotide (Figure 1bÁ, B́ and Ć). Thus, strong hybridization signals were observed in the cerebellum. Intermediate signals were detected over piriform cortex and, at a lesser extent, in hippocampus (CA1, CA3 and dentate gyrus) and some cortical areas such as entorhinal cortex (Figure 1bÁ, B́ and $C$ ). Using this technical approach, a significant increase in the munc18-1a mRNA labeling was observed in cerebellum and piriform and entorhinal cortex of Munc18-OE when compared with WT control animals (Figure 1c). Munc18-1a protein immunodensity increased in the brain cortex of Munc18-OE mice (Figure 1d) while munc18-1b did not change (Figure 1e). Enhanced munc18-1a protein immunofluorescence (Figure 1f) was found in cortex, hippocampus and cerebellum of the Munc18-OE mice (Figure 1g).

SNARE complex components. Neurotransmission processes are subjected to the control of exocytosis machinery, in which SNARE complex proteins play the key role. ${ }^{27}$ Syntaxin-1A, SNAP-25 and synaptobrevin, as well as synaptotagmin, did not change in the brain cortex of Munc18-OE mice. Stable heterotrimeric SNARE complexes in cortex showed no differences between Munc18-OE and WT controls (Supplementary Figure S2).

Schizophrenia-related phenotype. Munc18-OE and ageand sex-matched WT controls were subjected to a battery of tests for investigating schizophrenia-related behaviors. Munc18-OE mice spent less time exploring the center (Figure 2a) and more time the periphery (Figure 2b) of the open-field, consistent with an anxiety-like phenotype. In the novelty-induced hyperactivity test, the Munc18-OE mice exhibited increased locomotor activity during the first $5 \mathrm{~min}$, which is described as a schizophrenic-like behavior $^{15,28}$ (Figure 2c). No differences were found in the holeboard test for exploratory behavior (Supplementary Figure S3a) or in the rotarod test for motor coordination (Supplementary Figure S3b). In the elevated plus-maze test, Munc18-OE mice spent less time in the open-arms (Figure 2d) while decreased the number of entries to the open-arms (Figure 2e). These results reveal a high grade of aversion to unprotected spaces displayed by the Munc18-OE mice, which could mimic some of the negative symptoms of schizophrenia.

Deficits in social functioning represent a core negative symptom in schizophrenia and provide a quantifiable "negative symptom-like' behavior in animal models. ${ }^{14,15,29}$ Munc18OE mice showed a decrease in social interaction (Figure 2f). There were no changes in immobility time in the forced swim test in Munc18-OE mice (Supplementary Figure S3c), suggesting the absence of depressive-like symptoms.

Serotonin $5 \mathrm{HT}_{2 \mathrm{~A}}$ hallucinogenic drugs induce a quantifiable $\mathrm{HTR}$ in rodents that is not induced by non-hallucinogenic $5 \mathrm{HT}_{2 \mathrm{~A}}$ agonists. There are data supporting the HTR as a valid behavioral proxy of positive symptoms of psychosis in mice. ${ }^{30}$ Munc18-OE mice exhibited an increased HTR to administration of the hallucinogen $5 \mathrm{HT}_{2 \mathrm{~A}}$ agonist drug DOI (Figure $2 \mathrm{~g}$ ) when compared with WT controls.

Memory deficits in Munc18-OE mice were investigated by testing the animals on an episodic memory task for objects ('what'), their locations ('where') and the order in which they were experienced ('when') (Figure 2h). Munc18-OE mice 


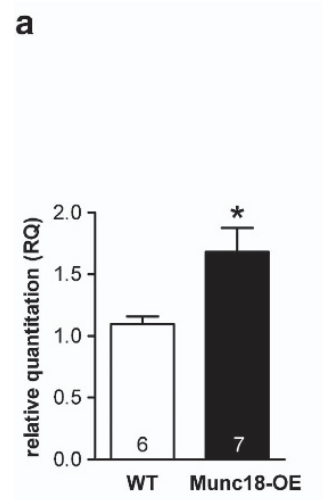

d

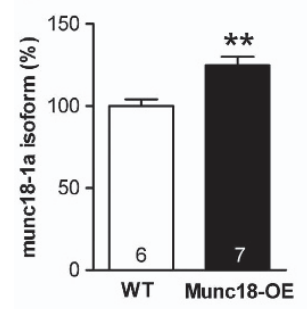

b

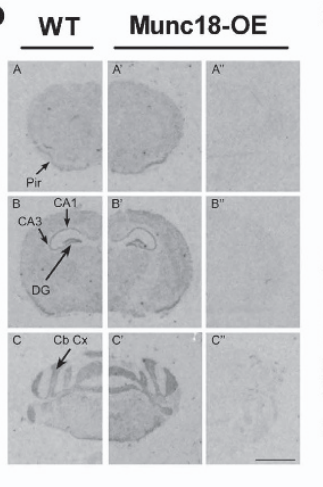

C

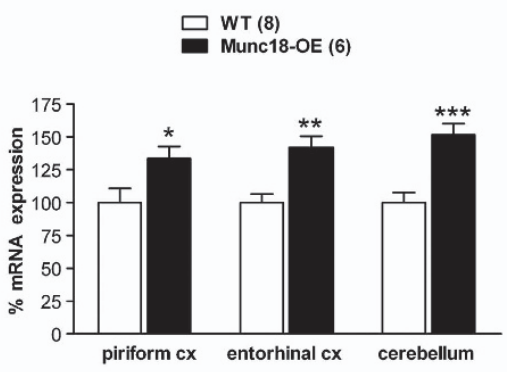

e

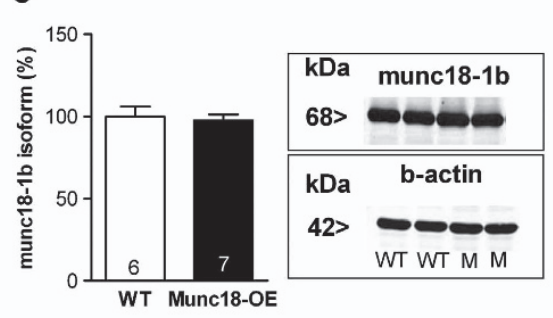

WT Munc18-OE
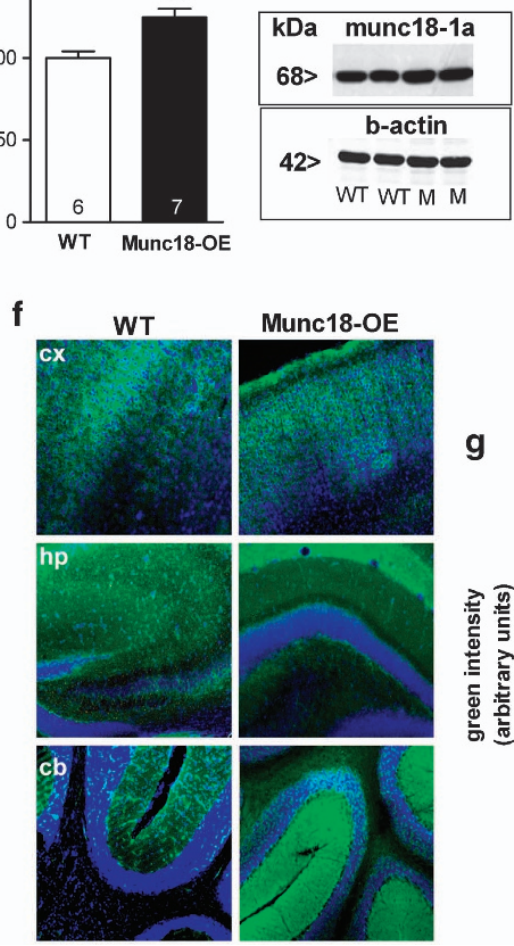

\section{Munc18-OE}

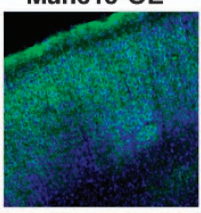

g
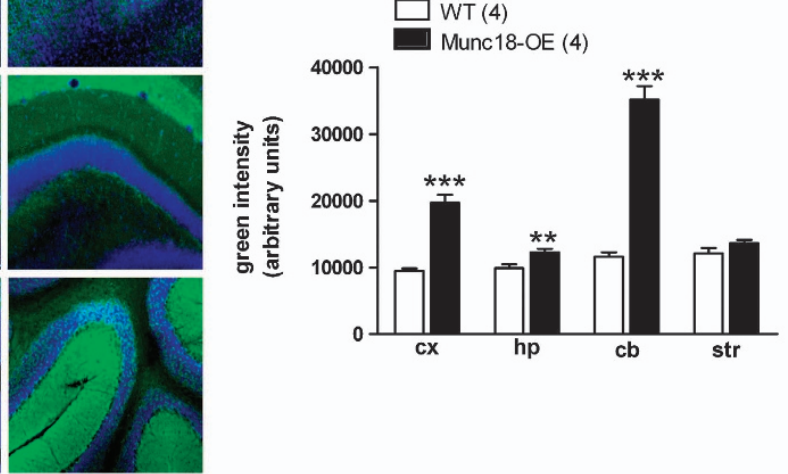

Figure 1 Characterization of Munc18-OE transgenic mice. (a) Quantitative real-time PCR study showed significant increased mRNA levels of munc18-1a in the brain cortex of Munc18-OE mice (unpaired $t$-test ${ }^{*} P<0.05$ versus wild-type (WT) controls). (b) Representative autoradiograms showing the distribution of munc18-1a mRNA in coronal, sections of WT controls (A, B, C) and transgenic Munc18 mice (A, B, Ć). The specificity labeling was demonstrated by the displacement with unlabeled oligonucleotide $(A, \dot{B}, \dot{C})$. Note the increase of mRNA signal in cerebellum and piriform cortex. Pir, piriform cortex; CA1, CA1 field of hippocampus; CA3, CA3 field of hippocampus; DG, dentate gyrus; $\mathrm{CB}$ Cx , Cerebellum cortex. Bar: $2 \mathrm{~mm}$. (c) Expression of mRNA encoding for munc18-1a in Munc18-OE and WT control mice evaluated by in situ hybridization. Significant increases of munc18-1a mRNA levels were obtained in different brain regions of Munc18-OE (unpaired $t$-test ${ }^{*} P<0.05$, ${ }^{* *} P<0.01$ and ${ }^{* * *} P<0.005$ versus WT control group). (d) Increased immunodensity of munc18-1a protein in the brain cortex of Munc18-OE mice (unpaired $t$-test ${ }^{* *} P<0.005$ versus WT controls). Representative immunoblots of munc18-1a and $\beta$-actin in WT controls and Munc18-OE mice. (e) As a negative control, no differences were found in munc18-1b protein expression between Munc18-OE and WT controls. (f) Representative images of munc18-1a distribution in WT (left panels) and Munc18-OE (right panels) at 10 $\times$, green (munc18-1a) and blue (nuclei) in cortex (cx), hippocampus (hp) and cerebellum (cb). (g) Increased immunofluorescence in different brain areas of Munc18-OE mice (unpaired $t$-test ${ }^{* *} P<0.005$ and ${ }^{\star \star \star} P<0.0001$ versus WT controls); cx, cortex; hp, hippocampus; $c b$, cerebellum; str, striatum. Data are mean \pm s.e.m.; the number of animals is indicated in parentheses or inside plot bars.

exhibited significant lower score in the memory for 'what'. Moreover, a significant decrease in score for 'where' memory was also found in Munc18-OE mice as well as in 'when' memory. These data suggest alterations in recognition memory processes, which have been described to be disrupted in schizophrenia. ${ }^{31}$ No differences were found in the Morris water-maze test (Supplementary Figure S3d), indicating that spatial memory is not affected in these transgenic animals.

Since PPI deficits have been reported in schizophrenia patients, ${ }^{32}$ Munc18-OE and WT mice were subjected to the PPI test in order to evaluate sensorimotor gating processes. 

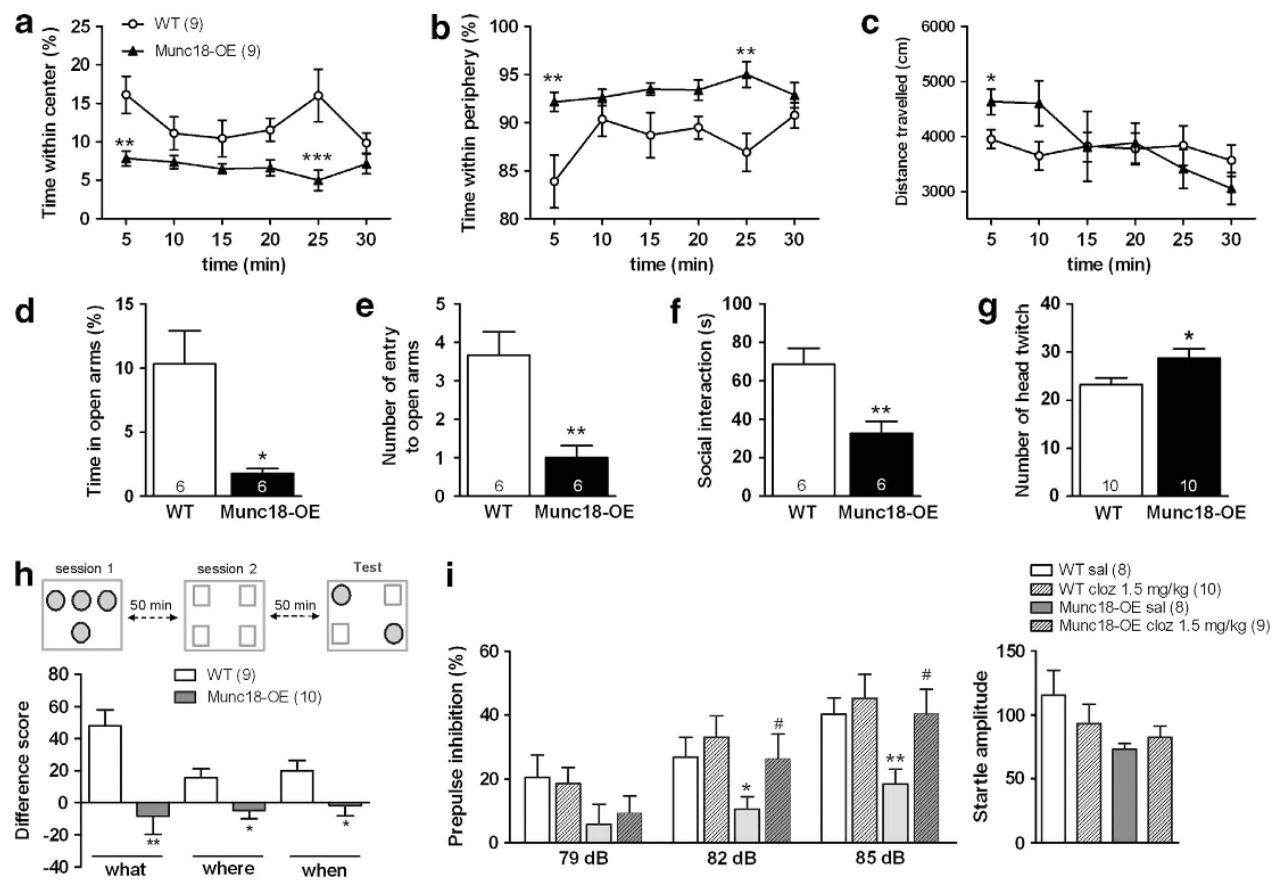

Figure 2 Overexpression of munc18-1a leads to schizophrenia-related behaviors. (a, b) Decreased central locomotion following exposure to a novel environment and increased exploration of the peripheral area in Munc18-OE transgenic mice (repeated-measures analysis of variance (ANOVA) followed by Bonferroni post hoc test, ${ }^{* *} P<0.01$ and ${ }^{* * *} P<0.001$ versus wild-type (WT) controls). (c) Increased locomotor activity of the transgenic mice during the first 5 min in novelty-induced hyperactivity test (unpaired $t$-test ${ }^{*} P<0.05$ versus WT controls). No differences between Munc18-OE and WT controls were found in total distance traveled in open field. (d) Munc18-OE mice displayed anxiety-like behavior in the elevated plus maze, as shown by the decreased time in open-arms (unpaired $t$-test ${ }^{*} P<0.05$ versus WT controls) and the decreased number of entries into open-arms (e) (unpaired $t$-test ${ }^{* *} P<0.01$ versus WT) (f) The social interaction test revealed decreased time of interaction elicited by transgenic Munc18OE mice (unpaired $t$-test ${ }^{\star \star} P<0.01$ versus WT controls). (g) Increased sensitivity to hallucinogenic-like action in Munc18-OE mice as shown by the increased number of headtwitch responses to DOI ( $1 \mathrm{mg} \mathrm{kg}^{-1}$, i.p.) administration (unpaired $t$-test ${ }^{\star} P<0.05$ versus WT controls). (h) Transgenic Munc18-OE mice exhibited altered episodic memory as shown in the novel-object recognition test. Decreased score in memory for objects (what), their locations (where), and the order in which they were experienced (when) (unpaired $t$-test ${ }^{*} P<0.05$ and ${ }^{*} P<0.005$ versus WT controls). (i) Munc18-OE mice showed impaired prepulse inhibition (PPI) of the acoustic startle reflex across prepulse intensities (two-way analysis of variance (ANOVA) ${ }^{\star * \star} P<0.001$ for genotype factor; unpaired $t$-test ${ }^{\star} P<0.05$ and ${ }^{* \star} P<0.005$ versus WT controls). Impairment of the PPI was completely reversed in the transgenic mice after clozapine ( $1.5 \mathrm{mg} \mathrm{kg}^{-1}$ i.p.) administration (unpaired $t$-test ${ }^{\#} P<0.05$ versus transgenic animals with saline). No differences were found in the startle amplitude between Munc18-OE and WT controls. Data are mean \pm s.e.m.; the number of animals is indicated in parentheses or inside the plot bars.

Munc18-OE mice exhibited a significant decrease in the PPI of the acoustic startle reflex at different intensities (Figure 2i), while no differences were found in the startle amplitude between transgenic Munc18-OE and WT controls. Acute clozapine $\left(1.5 \mathrm{mg} \mathrm{kg}^{-1}\right.$ i.p.) reversed the PPI deficits in Munc18-OE mice while exerted no changes in WT controls (Figure 2i).

Dopaminergic system alterations. DA signaling dysregulation has been proposed as a crucial component of the pathophysiology of schizophrenia. ${ }^{2} D_{1}$ receptor density decreased in both cortex and striatum of Munc18-OE mice (Figure 3a) while $D_{2}$ receptor density did not change (Figure $3 b$ ). Similar alterations of $D_{1}$ and integral components of its signal transduction pathways have been found in patients with schizophrenia. ${ }^{33,34}$ The DA membrane transporter (DAT) is a critical regulator of DA neurotransmission in the brain. Due to the difficulty of DAT immunodetection in cortical regions, striatal DAT was evaluated and a strong decrease was found in Munc18-OE mice (Figure 3c). These data are in accordance with those studies reporting an in vivo decrease of DAT in patients with schizophrenia. ${ }^{35}$ This decrease of DAT suggests that intracellular pools of DA would probably be altered. Therefore, TH (tyrosine hydroxylase) was evaluated and results showed increased density in both cortex and striatum of Munc18-OE mice (Figure 3d). Basal extracellular concentrations of DA in both regions were no different between Munc18-OE mice and their controls. However, administration of a single dose of amphetamine $\left(5 \mathrm{mg} \mathrm{kg}^{-1}\right.$ i.p.) induced an enhanced locomotor hyperactivity (Figure $3 e$ ) and higher increase of extracellular DA in both prefrontal cortex (Figure 3f) and striatum (Figure $3 \mathrm{~g}$ ) of Munc18-OE when compared with WT controls. Similar supersensitivity to amphetamine-induced increase of DA release has been described in schizophrenic patients when evaluated by in vivo neuroimaging. ${ }^{36}$

Glutamate receptors. Abnormalities in glutamate neurotransmission have been implicated in major psychiatric disorders such as schizophrenia. ${ }^{37}$ No changes of mGluR2 and mGluR3 receptors (Supplementary Figure S4a), AMPAR1, AMPAR2/3/4 (Supplementary Figure S4b), NR1 and NR2A (Supplementary Figure S4c) protein subunits were found in the brain cortex of Munc18-OE mice. No changes of the glutamate signaling activation protein PSD95 were observed (Supplementary Figure S4d). Src, a kinase 

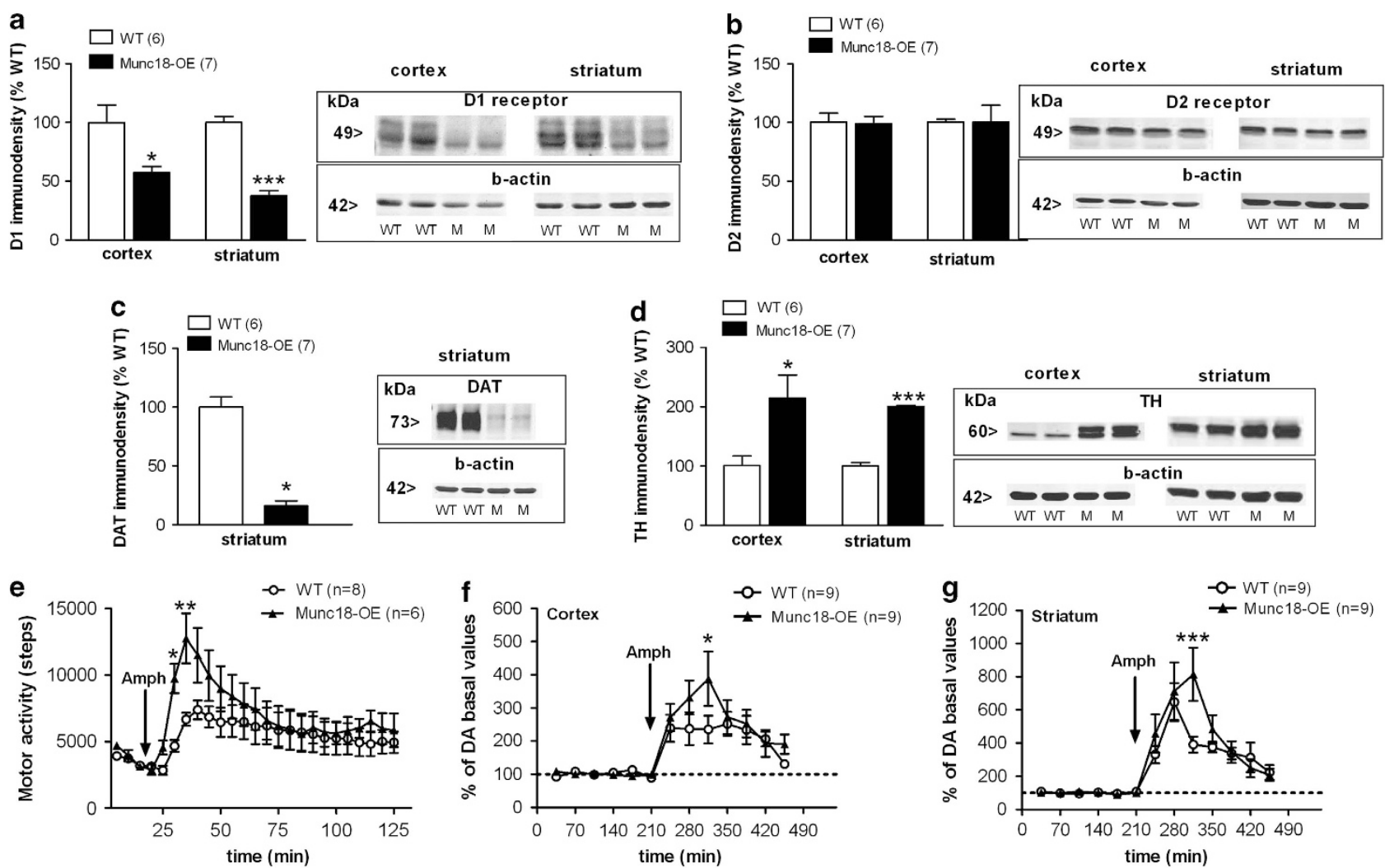

Figure 3 Alterations of dopaminergic system components. (a) Munc18-OE mice displayed decreased dopamine $D_{1}$ receptor density versus wild-type (WT) controls in both cortical and striatal brain regions (unpaired $t$-test ${ }^{*} P<0.05$ and ${ }^{*} P<0.0001$ versus WT controls). Representative immunoblots of dopamine $D_{1}$ receptor and $\beta$-actin in WT controls and Munc18-OE mice. (b) No differences were observed in dopamine $D_{2}$ receptor immunodensity between transgenic Munc18-OE and WT control mice. (c) Striatal dopamine transporter (DAT) was strongly decreased in Munc18-OE mice (unpaired $t$-test ${ }^{*} P<0.001$ versus WT controls). Representative immunoblots of DAT and $\beta$-actin in WT controls and Munc18-OE mice. (d) Munc18-OE mice exhibited an increase of the dopamine synthesizing enzyme tyrosine hydroxylase (TH) in both cortex and striatum (unpaired $t$-test ${ }^{*} P<0.05$ and ${ }^{* \star *} P<0.0001$ versus WT controls). Data bars of immunoblots are mean (percentage over controls) \pm s.e.m.; the number of animals is indicated in parentheses. (e) Increased motor activity in Munc18-OE mice $(\boldsymbol{\Delta})$ versus WT controls $(O)$ following a single injection of $5 \mathrm{mg} \mathrm{kg}^{-1}$ i.p. of amphetamine (Amph) (repeatedmeasures two-way analysis of variance (ANOVA) followed by Bonferroni post hoc test, ${ }^{*} P<0.05$ and ${ }^{* *} P<0.01$ versus WT). (f, g) Effect of Amph (5 mg kg ${ }^{-1}$ i.p.) on extracellular DA concentrations in the prefrontal cortex (f) or striatum $(\mathbf{g})$ of Munc18-OE mice $(\mathbf{O})$ and their WT controls $(\bigcirc)$ expressed as percentage of DA basal concentration values (two-way ANOVA followed by Bonferroni post hoc test, ${ }^{*} P<0.05$ and ${ }^{* * *} P<0.001$ versus WT controls). The mean of the six first samples were considered basal values and assumed as the $100 \%$ value. Values were expressed as percentage of the basal values. Points are mean \pm s.e.m.; the number of animals is indicated in parentheses.

that phosphorylates and subsequently activates NR2A subunits, was also evaluated and no differences were found (Supplementary Figure S4e).

In vivo volumetric study of gray matter loss. Numerous findings from imaging studies strongly support that decrements of gray matter volume are involved in schizophrenia. ${ }^{3,4}$ The magnetic resonance imaging (MRI) volumetric study revealed a significant reduction of gray matter volume in Munc18-OE mice (Supplementary Table S2) in right and left temporal cortex (Figure 4a), prefrontal-olfactory cortex (Figure 4b) and cerebellar lobe (Figure 4c).

Changes in apoptotic and inflammatory markers. Several studies have suggested a role of apoptotic pathways in the cortical brain volume deficits in schizophrenia. ${ }^{38} \mathrm{Fas}$ receptor showed no changes in Munc18-OE mice (Figure 5a), FADD protein increased and no changes were observed in p-Ser191-FADD (Figure 5b). Caspase-3 activity (Figure 5c) and PARP-1 density did not change in Munc18-
OE (Figure 5d). Oxidative insults are well-established triggers of apoptosis and oxidative stress has been implicated in the pathophysiology of schizophrenia. ${ }^{39}$ Munc18-OE exhibited an increase of inducible nitric oxide synthase (NOS2) density in the brain cortex whereas no changes were observed in cyclooxygenase 2 (COX2) or in neuronal nitric oxide synthase (NOS1) (Figure 5e). Malondialdehyde (MDA) cortical levels were quantified to test whether lipid peroxidation could be altered in Munc18-OE animals although no changes were found (Figure $5 f$ ).

\section{Discussion}

This study demonstrates that munc18-1 is involved in the development of behavioral, neurochemical and morphological changes that resemble those observed in schizophrenia. Munc18-OE mice seem to fulfill the validity requirements for an animal model of schizophrenia. ${ }^{14,15,28}$ Thus, the transgenic Munc18-OE animal: (a) was developed on the basis of precvious findings in human brain of subjects with 
schizophrenia ${ }^{10,11}$ (construct validity); (b) exhibits schizophrenia-related phenotypes (face validity); and (c) normalizes PPI deficits following antipsychotic drug treatment (predictive
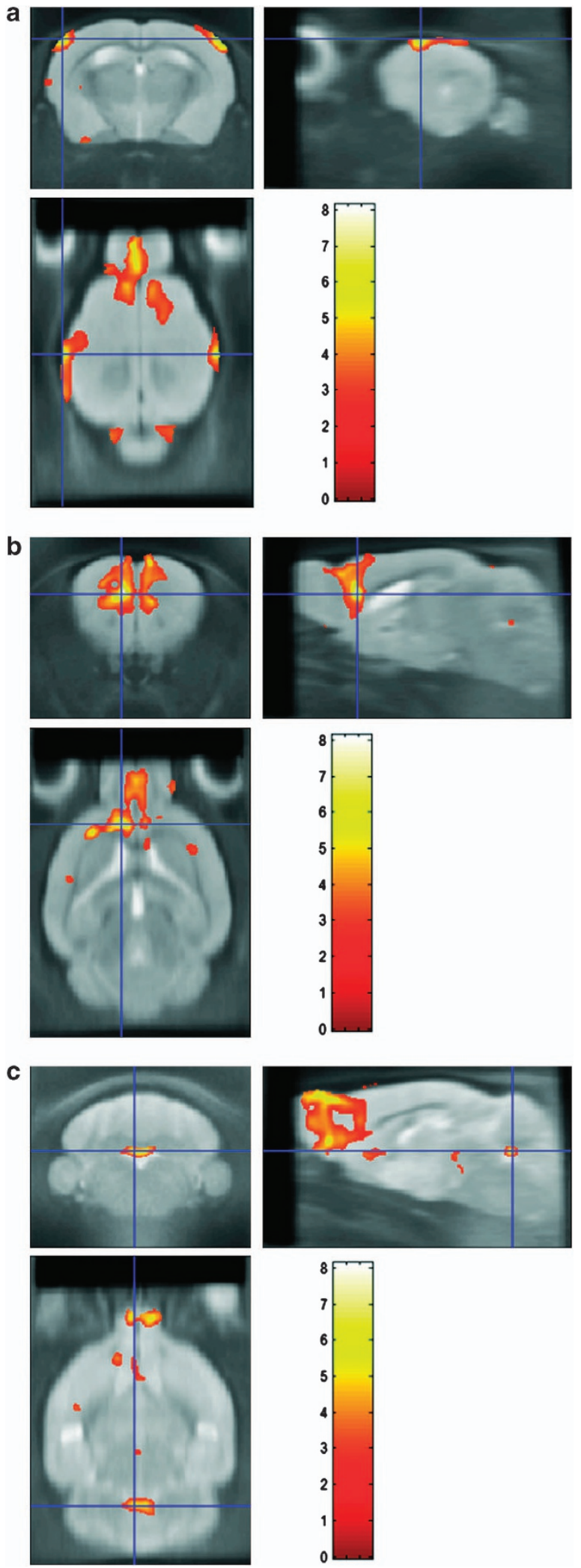

validity). Furthermore, the present study strongly supports the hypothesis that abnormalities in exocytosis play a key role in the pathophysiology of schizophrenia.

Dysregulation of munc18-1 in postmortem brain of schizophrenic subjects is still controversial. Thus, increased expression in membrane microdomains, ${ }^{10}$ unaltered expression in total protein ${ }^{11}$ and decreased coimmunoprecipitation with syntaxin- 1 of munc $18-1^{9}$ have been described in the brain of schizophrenic subjects. The Munc18-OE mouse design was based on the overexpression of the munc18-1 protein $^{10}$ and the downregulation exerted by antipsychotics ${ }^{11}$ in human brain.

Overexpression of munc18-1 induces a spectrum of behaviors that correlates with symptoms observed in schizophrenia. $^{28}$ This model displayed anxiety-like behaviors and aversion to unprotected new situations as observed in novelty-induced hyperlocomotion or elevated plus-maze tests. It has been described that patients with schizophrenia often develop additional comorbid conditions, including anxiety disorders. ${ }^{40}$ On the other hand, animals overexpressing munc18-1 did not present a depressive-like behavior, at least in the test used in this study for evaluation of depressive behaviors. Deficits in social functioning, which represent a core negative symptom in schizophrenia, ${ }^{29}$ were also present in these transgenic mice as revealed the decreased social interaction. Moreover, these mice displayed cognitive alterations that resemble cognitive deficits observed in schizophrenia. ${ }^{40}$

The PPI deficits observed in Munc18-OE mice evidenced the existence of profound alterations in sensorimotor gating processes. PPI test has been considered as an excellent tool for studying the neural basis of schizophrenia due to the relevance of deficits in filtering sensorial and cognitive information in the pathophysiology of this disorder. ${ }^{32}$ The fact that the atypical antipsychotic clozapine completely reversed the gating deficit strongly supports the Munc18-OE as an animal model that mimics schizophrenia. In addition, the increase in hallucinogenic-like response susceptibility, as demonstrated the effects of $\mathrm{DO}$ administration, might indicate that Munc18-OE is a good model for this positive core symptom of psychosis and schizophrenia.

DA signaling dysregulation has been proposed as a crucial component of the pathogenesis of schizophrenia ${ }^{2}$ and it has been suggested that abnormal cognitive functioning in schizophrenia is related to altered DA function in the prefrontal cortex. ${ }^{33}$

The Munc18-OE mice showed a decrease of $D_{1}$ without changes of $D_{2}$ receptor protein in both brain cortex and striatum. Studies in humans have shown controversy about $D_{1}$ receptor in schizophrenia. Some binding studies have reported decreased prefrontal $D_{1}$ receptors in

Figure 4 Volumetric reduction in gray matter of Munc18-OE transgenic mice. (a) A reduction in volume in temporal cortex was observed, $P<0.01, K=200$ voxels, $T=5.66$ (left side) and 6.02 (right side). (b) A reduction in volume in prefrontal-olfatory-cingulate cortex was shown, $P<0.01, K=200$ voxels, $T=8.11$, as well as in (c) cerebellar lobe $P<0.01, K=200$ voxels, $T=5.25$. Images were analyzed according to voxel-based morphometry (VBM) methodology (see Materials and methods for details). Color bar indicates potency of significance level, from 0 (less potency) to 8 (more potency). 
a

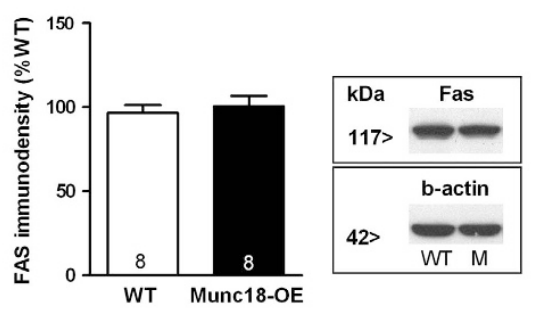

d

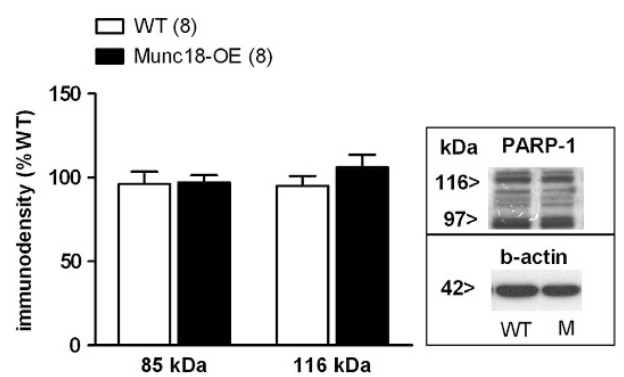

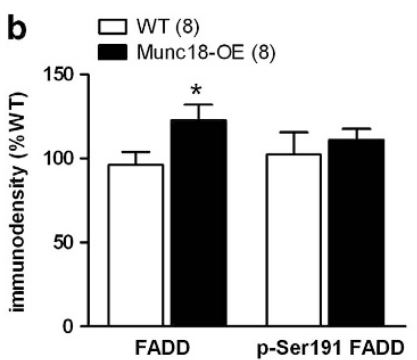

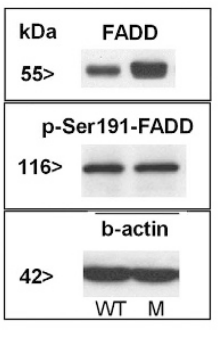

C

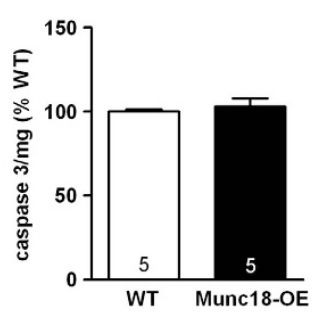

e

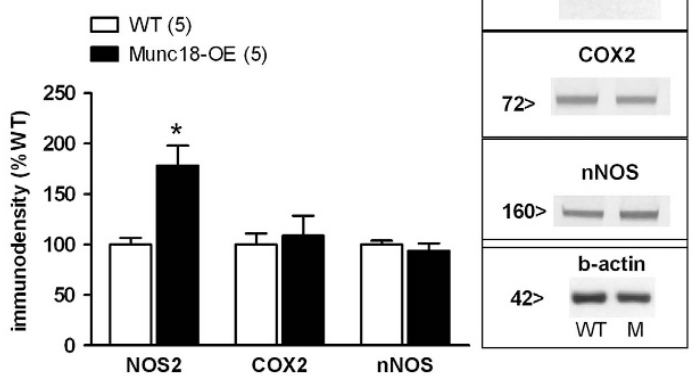

f

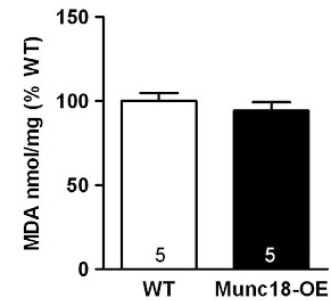

Figure 5 Apoptotic pathways and inflammatory markers. (a) No changes in Fas receptor density (glycosylated form), evaluated by western blot, were found in cortex of Munc18-OE mice. Representative immunoblots of Fas receptor and $\beta$-actin in WT controls and Munc18-OE mice (b) FADD (dimeric form) significantly increased in Munc18OE mice (unpaired $t$-test ${ }^{*} P<0.05$ versus WT controls) while phosphorylated $p$-191 FADD (oligomeric form) remained unchanged. Representative immunoblots of FADD, p-191 FADD and $\beta$-actin in WT controls and Munc18-OE mice. (c) No changes were found in caspase-3 activity. (d) No aberrant changes were observed in the proteolytic cleavage of nuclear PARP-1. Representative immunoblots of PARP-1, 85 fragment and $\beta$-actin in WT and Munc18-OE mice. (e) An increase of inducible nitric oxide synthase (NOS2) was detected in the brain cortex of Munc18-OE (unpaired $t$-test ${ }^{\star} P<0.05$ versus WT controls). The Munc18-OE mice did not present changes in the pro-inflammatory enzyme ciclooxygenase 2 (COX2) and in neuronal nitric oxide synthase (NOS1). Representative immunoblots of NOS2/COX2/NOS1 and $\beta$-actin in WT controls and Munc18OE mice. (f) Lipid peroxidation was studied by measuring the levels of malondialdehyde (MDA) in the brain cortex of Munc19-OE and WT controls. No changes were found in cortical MDA of Munc18-OE mice when compared with their WT controls. Data bars are mean (percentage over controls) \pm s.e.m.; the number of animals is indicated in parentheses or inside the plot bars.

schizophrenia, ${ }^{34,41}$ while others have found no changes ${ }^{42}$ or even an increase in $D_{1}$ binding potential. ${ }^{43}$ Increased binding of $D_{1}$ does not necessary represent an overexpression of the receptor, since it might be due to an increase in receptor affinity. ${ }^{44}$ Despite these confusing data, it has been postulated that both upregulation and downregulation of $D_{1}$ receptors in the prefrontal cortex are closely related to cognitive symptoms. ${ }^{33,43} D_{2}$ receptors are widely expressed in striatal areas and their regulation in schizophrenia is also a focus of discussion. Imaging studies have found no changes of $D_{2}$ receptors in treatment-naive patients with schizophrenia, ${ }^{45}$ while in post-mortem human tissue it has been reported an increase of $D_{2}$ receptor in striatal areas ${ }^{44}$ or even no changes in prefrontal cortex. ${ }^{19}$ Moreover, the relationship between increases of $D_{2}$ receptors and exposure to antipsychotics is not still well established and $D_{2}$ upregulation in schizophrenia might be induced by neuroleptic long-term treatment. ${ }^{44,46}$

The DA membrane transporter (DAT) is a critical regulator of dopaminergic neurotransmission in the brain. In this animal model, overexpression of munc18-1a led to a strong downregulation of DAT in striatum. As occurs with DA receptors, also the status of DAT in the brain of subjects with schizophrenia remains unclear. However, DAT knockout mice have provided an opportunity to model in vivo conditions of extreme dopaminergic dysfunction. ${ }^{47}$ DAT knockout mice exhibit impairment in social interaction ${ }^{48}$ and deficits in PPI that are attenuated after antipsychotic administration. ${ }^{49}$ It has been postulated that in the absence of DAT, extracellular DA clearance from synaptic cleft slows down, which leads to the downregulation of DA receptors. ${ }^{47}$ The observed strong decrease of DAT in Munc18-OE might contribute to gating deficits and social dysfunction. $\mathrm{TH}$ was strongly overexpressed in cortex and striatum of Munc18-OE. Equivalent TH overexpression has been demonstrated under conditions of chronic blockade of DAT by cocaine administration. ${ }^{50}$ Because of the $D_{1}$ receptor abnormalities, the reduction of presynaptic DAT and the overexpression of $\mathrm{TH}$, it is tempting to speculate the presence of hyperdopaminergia in the brain of Munc18-OE mice. Hyperactivity of presynaptic DA terminals has been proposed to be a key component of schizophrenia ${ }^{2,51}$ and should be reflected in alteration of extracellular DA concentrations under in vivo conditions. Although basal DA concentrations in the brain cortex and striatum of Munc18-OE were not modified, amphetamine challenge promoted an enhanced release of DA when compared with controls. Similar findings have been reported by in vivo neuroimaging in patients with schizophrenia. ${ }^{36}$ Moreover, the enhanced sensitivity of extracellular DA to amphetamine administration in Munc18-OE is concordant with the hyperlocomotion observed in these animals in response to amphetamine. In fact, this hyperactivity has been proposed as a valuable model for psychosis and positive symptoms of schizophrenia. All these data reveal that the dopaminergic dysfunction in Munc18-OE animals might be 
representative of the spectrum of symptoms observed in humans with schizophrenia.

Imaging studies suggest that schizophrenia is a brain disease involving decrements of gray matter volume. ${ }^{3,4}$ The significant decrease of gray matter in temporal and prefrontalolfactory cortex as well as in cerebellar lobe of Munc18-OE when evaluated by MRI revealed the importance of this model for the study of the schizophrenia pathogenesis. Although mechanisms underlying the decrease of cortical brain volume in schizophrenia are unknown, it could suggest a role of apoptotic pathways in these deficits. ${ }^{38}$ Changes of apoptotic pathways in cortex of Munc18-OE mice were scarce but a proapoptotic trend in apoptosis pathways and susceptibility to neural damage induced by elevated NOS2 might be contributing to the reported decreases of gray matter. Further studies beyond this exploratory analysis of the Munc18-OE phenotype are necessary to establish the mechanisms leading from munc18-1a overexpression to regional brain loss of gray matter.

Since schizophrenia is a disorder with non-well understood origin, pathogenesis, and pathophysiology, animal models could contribute to decipher the key components of the disease and its response to treatment. In this context, the present study demonstrates that dysfunctions of munc18-1 are relevant for the development of a wide spectrum of behavioral, neurochemical and anatomical alterations that may resemble schizophrenic symptoms and/or neurobiological findings observed in subjects with schizophrenia.

\section{Conflict of interest}

LU, MJG, MF-A and JJM are authors of a patent (WO/2010/ 020642) related to munc18-1 in schizophrenia owned by Brainco Biopharma SL and UPV/EHU. JJM and AP have received research funds from Brainco Biopharma SL.

Acknowledgements. We thank Raquel Rey-Brea, Alfredo Ramos-Miguel and Nagore Puente for technical assistance. The study was supported by an intramural Grant from Centro de Investigación Biomédica en Red de Salud Mental, Instituto de Salud Carlos III (CIBERSAM), Spanish MICINN and FEDER (SAF200908460 to JJM; SAF2010-21948 to JLMMM; AGL2009-11358 to AG-A, SAF2011. 29918 to JAGS; PI10/02986, CP08/00017 and CEN-20101014 to MD), Basque Government (S-PR10UN01 to JEO and IT-199/07 to JJM), University of the Basque Country (UPV/EHU) and Complutense University of Madrid (UCM GR42/10-962075 to JLMM).

1. van Os J, Kapur S. Schizophrenia. Lancet 2009; 374: 635-645

2. Howes OD, Kapur S. The dopamine hypothesis of schizophrenia: version III-the final common pathway. Schizophr Bull 2009; 35: 549-562

3. Honea R, Crow TJ, Passingham D, Mackay CE. Regional deficits in brain volume in schizophrenia: a meta-analysis of voxel-based morphometry studies. Am J Psychiatry 2005; 162: 2233-2245

4. Arango C, Rapado-Castro M, Reig S, Castro-Fornieles J, Gonzalez-Pinto A, Otero S et al. Progressive brain changes in children and adolescents with first-episode psychosis. Arch Gen Psychiatry 2012; 69: 16-26.

5. Johnson RD, Oliver PL, Davies KE. SNARE proteins and schizophrenia: linking synaptic and neurodevelopmental hypotheses. Acta Biochim Pol 2008; 55: 619-628.

6. Sudhof TC, Rothman JE. Membrane fusion: grappling with SNARE and SM proteins. Science 2009; 323: 474-477.

7. Burgoyne RD, Barclay JW, Ciufo LF, Graham ME, Handley MT, Morgan A. The functions of Munc18-1 in regulated exocytosis. Ann NY Acad Sci 2009; 1152: 76-86.

8. Toonen RF, Verhage M. Munc18-1 in secretion: lonely Munc joins SNARE team and takes control. Trends Neurosci 2007; 30: 564-572.
9. Castillo MA, Ghose S, Tamminga CA, Ulery-Reynolds PG. Deficits in syntaxin 1 phosphorylation in schizophrenia prefrontal cortex. Biol Psychiatry 2010; 67: 208-216

10. Behan AT, Byrne C, Dunn MJ, Cagney G, Cotter DR. Proteomic analysis of membrane microdomain-associated proteins in the dorsolateral prefrontal cortex in schizophrenia and bipolar disorder reveals alterations in LAMP, STXBP1 and BASP1 protein expression. $\mathrm{Mol}$ Psychiatry 2009; 14: 601-613.

11. Gil-Pisa I, Munarriz-Cuezva E, Ramos-Miguel A, Uriguen L, Meana JJ, Garcia-Sevilla JA. Regulation of munc18-1 and syntaxin-1A interactive partners in schizophrenia prefrontal cortex: down-regulation of munc18-1a isoform and $75 \mathrm{kDa}$ SNARE complex after antipsychotic treatment. Int J Neuropsychopharmacol 2012; 14: 573-588.

12. Garcia EP, McPherson PS, Chilcote TJ, Takei K, De Camilli P. rbSec1A and B colocalize with syntaxin 1 and SNAP-25 throughout the axon, but are not in a stable complex with syntaxin. J Cell Biol 1995; 129: 105-120.

13. Swanson DA, Steel JM, Valle D. Identification and characterization of the human ortholog of rat STXBP1, a protein implicated in vesicle trafficking and neurotransmitter release. Genomics 1998; 48: 373-376.

14. Nestler EJ, Hyman SE. Animal models of neuropsychiatric disorders. Nat Neurosci 2010; 13: $1161-1169$.

15. Powell CM, Miyakawa T. Schizophrenia-relevant behavioral testing in rodent models: a uniquely human disorder? Biol Psychiatry 2006; 59: 1198-1207.

16. Gutierrez-Adan A, Pintado B. Effect of flanking matrix attachment regions on the expression of microinjected transgenes during preimplantation development of mouse embryos. Transgenic Res 2000; 9: 81-89.

17. Garcia-Sevilla JA, Alvaro-Bartolome M, Diez-Alarcia R, Ramos-Miguel A, Puigdemont D, Perez $V$ et al. Reduced platelet $G$ protein-coupled receptor kinase 2 in major depressive disorder: antidepressant treatment-induced upregulation of GRK2 protein discriminates between responder and non-responder patients. Eur Neuropsychopharmacol 2010; 20: 721-730.

18. Vaidya VA, Castro ME, Pei Q, Sprakes ME, Grahame-Smith DG. Influence of thyroid hormone on $5-\mathrm{HT}(1 \mathrm{~A})$ and $5-\mathrm{HT}(2 \mathrm{~A})$ receptor-mediated regulation of hippocampal BDNF mRNA expression. Neuropharmacology 2001; 40: 48-56.

19. Urigüen L, Garcia-Fuster MJ, Callado LF, Morentin B, La Harpe R, Casado V et al. Immunodensity and mRNA expression of A2A adenosine, D2 dopamine, and CB1 cannabinoid receptors in postmortem frontal cortex of subjects with schizophrenia: effect of antipsychotic treatment. Psychopharmacology 2009; 206: 313-324.

20. Gonzalez-Maeso J, Ang RL, Yuen T, Chan P, Weisstaub NV, Lopez-Gimenez JF et al. Identification of a serotonin/glutamate receptor complex implicated in psychosis. Nature 2008; 452: 93-97.

21. Uriguen L, Perez-Rial S, Ledent C, Palomo T, Manzanares J. Impaired action of anxiolytic drugs in mice deficient in cannabinoid CB1 receptors. Neuropharmacology 2004; 46: 966-973.

22. Paxinos G, Franklin KBJ. The Mouse Brain in Stereotaxic Coordinates. Academic Press: San Diego, 2001.

23. Ortega JE, Fernandez-Pastor B, Callado LF, Meana JJ. In vivo potentiation of reboxetine and citalopram effect on extracellular noradrenaline in rat brain by alpha(2)-adrenoceptor antagonism. Eur Neuropsychopharmacol 2010; 20: 813-822.

24. Sawiak SJ, Wood NI, Williams GB, Morton AJ, Carpenter TA. Voxel-based morphometry in the $\mathrm{R} 6 / 2$ transgenic mouse reveals differences between genotypes not seen with manual 2D morphometry. Neurobiol Dis 2009; 33: 20-27.

25. Conti F, DeBiasi S, Minelli A, Rothstein JD, Melone M. EAAC1, a high-affinity glutamate tranporter, is localized to astrocytes and gabaergic neurons besides pyramidal cells in the rat cerebral cortex. Cereb Cortex 1998; 8: 108-116.

26. Rothstein JD, Dykes-Hoberg M, Pardo CA, Bristol LA, Jin L, Kuncl RW et al. Knockout of glutamate transporters reveals a major role for astroglial transport in excitotoxicity and clearance of glutamate. Neuron 1996; 16: 675-686.

27. Rizo J, Sudhof TC. Snares and Munc18 in synaptic vesicle fusion. Nat Rev Neurosci 2002; 3: $641-653$

28. Arguello PA, Gogos JA. Modeling madness in mice: one piece at a time. Neuron 2006; 52 : 179-196

29. O'Tuathaigh CM, Kirby BP, Moran PM, Waddington JL. Mutant mouse models: genotypephenotype relationships to negative symptoms in schizophrenia. Schizophr Bull 2010; 36: 271-288.

30. Gonzalez-Maeso J, Weisstaub NV, Zhou M, Chan P, Ivic L, Ang R et al. Hallucinogens recruit specific cortical $5-\mathrm{HT}(2 \mathrm{~A})$ receptor-mediated signaling pathways to affect behavior. Neuron 2007; 53: 439-452.

31. Aleman A, Hijman R, de Haan EH, Kahn RS. Memory impairment in schizophrenia: a metaanalysis. Am J Psychiatry 1999; 156: 1358-1366.

32. Braff DL, Geyer MA, Swerdlow NR. Human studies of prepulse inhibition of startle: normal subjects, patient groups, and pharmacological studies. Psychopharmacology 2001; 156: 234-258.

33. Goldman-Rakic PS, Castner SA, Svensson TH, Siever LJ, Williams GV. Targeting the dopamine D1 receptor in schizophrenia: insights for cognitive dysfunction. Psychopharmacology (Berl) 2004; 174: 3-16.

34. Okubo Y, Suhara T, Suzuki K, Kobayashi K, Inoue O, Terasaki O et al. Decreased prefrontal dopamine D1 receptors in schizophrenia revealed by PET. Nature 1997; 385 634-636. 
35. Laakso A, Bergman J, Haaparanta M, Vilkman H, Solin O, Syvalahti E et al. Decreased striatal dopamine transporter binding in vivo in chronic schizophrenia. Schizophr Res 2001; 52: $115-120$.

36. Laruelle M, Abi-Dargham A, van Dyck CH, Gil R, D'Souza CD, Erdos J et al. Single photon emission computerized tomography imaging of amphetamine-induced dopamine release in drug-free schizophrenic subjects. Proc Natl Acad Sci USA 1996; 93: 9235-9240.

37. Tamminga C. Glutamatergic aspects of schizophrenia. Br J Psychiatry Suppl 1999; 37: 12-15.

38. Glantz LA, Gilmore JH, Lieberman JA, Jarskog LF. Apoptotic mechanisms and the synaptic pathology of schizophrenia. Schizophr Res 2006; 81: 47-63.

39. Bernstein HG, Bogerts B, Keilhoff G. The many faces of nitric oxide in schizophrenia. A review. Schizophr Res 2005; 78: 69-86.

40. Buckley PF, Miller BJ, Lehrer DS, Castle DJ. Psychiatric comorbidities and schizophrenia Schizophr Bull 2009; 35: 383-402.

41. Kosaka J, Takahashi $\mathrm{H}$, Ito $\mathrm{H}$, Takano A, Fujimura $\mathrm{Y}$, Matsumoto $\mathrm{R}$ et al. Decreased binding of [11C]NNC112 and [11C]SCH23390 in patients with chronic schizophrenia. Life Sci 2010; 86: 814-818.

42. Karlsson P, Farde L, Halldin C, Sedvall G. PET study of D(1) dopamine receptor binding in neuroleptic-naive patients with schizophrenia. Am J Psychiatry 2002; 159: 761-767.

43. Abi-Dargham A, Mawlawi O, Lombardo I, Gil R, Martinez D, Huang $Y$ et al. Prefrontal dopamine D1 receptors and working memory in schizophrenia. J Neurosci 2002; 22: 3708-3719.

44. Seeman P. Targeting the dopamine D2 receptor in schizophrenia. Expert Opin Ther Targets 2006; 10: 515-531.

45. Guillin O, Abi-Dargham A, Laruelle M. Neurobiology of dopamine in schizophrenia. Int Rev Neurobiol 2007; 78: 1-39.
46. Silvestri S, Seeman MV, Negrete JC, Houle S, Shammi CM, Remington GJ et al. Increased dopamine D2 receptor binding after long-term treatment with antipsychotics in humans: a clinical PET study. Psychopharmacology 2000; 152: 174-180.

47. Giros B, Jaber M, Jones SR, Wightman RM, Caron MG. Hyperlocomotion and indifference to cocaine and amphetamine in mice lacking the dopamine transporter. Nature 1996; 379 : 606-612.

48. Rodriguiz RM, Chu R, Caron MG, Wetsel WC. Aberrant responses in social interaction of dopamine transporter knockout mice. Behav Brain Res 2004; vol. 148: 185-198.

49. Powell SB, Young JW, Ong JC, Caron MG, Geyer MA. Atypical antipsychotics clozapine and quetiapine attenuate prepulse inhibition deficits in dopamine transporter knockout mice. Behav Pharmacol 2008; 19: 562-565.

50. Vrana SL, Vrana KE, Koves TR, Smith JE, Dworkin SI. Chronic cocaine administration increases CNS tyrosine hydroxylase enzyme activity and mRNA levels and tryptophan hydroxylase enzyme activity levels. J Neurochem 1993; 61: 2262-2268.

51. Howes OD, Kambeitz J, Kim E, Stahl D, Slifstein M, Abi-Dargham A et al. The nature of dopamine dysfunction in schizophrenia and what this means for treatment. Arch Gen Psychiatry 2012; 69: 776-786.

Translational Psychiatry is an open-access journal published by Nature Publishing Group. This work is licensed under the Creative Commons Attribution-NonCommercial-No Derivative Works 3.0 Unported License. To view a copy of this license, visit http://creativecommons.org/licenses/by-nc-nd/3.0/

Supplementary Information accompanies the paper on the Translational Psychiatry website (http://www.nature.com/tp) 